\title{
ATMOSPHERICISPACE ENVIRONMENT SUPPORT LESSONS LEARNED REGARDING AEROSPACE VEHICLE DESIGN AND OPERATIONS
}

\author{
William W. Vaughan* \\ University of Alabama in Huntsville \\ B. Jeffrey Anderson \\ NASA Marshall Space Flight Center
}

\section{INTRODUCTION}

Organized atmospheric and space environment support for aerospace vehicle design and operations came into being in the 1950s as the development of large rockets for military and civilian usage grew in the United States. This support focused on the development of natural environment models, design/operational requirements, and environment measurement systems to support the needs of aerospace vehicles, both launch vehicles and spacecraft. It encompassed the launch, ascent, and entry flight environment regions, including Earth orbit environments. Several groups within the United States were active in this area, including the Department of Defense, National Aeronautics and Space Administration, and a few in the aerospace industry. Some atmospheric environment support efforts were similar to those being undertaken for aviation interests. As part of the atmospheric and space environment support activities a number of lessons learned resulted. These produced follow on efforts that benefited from these experiences. These lead to the rather efficient and technologically current atmospheric and space environment descriptions, design requirements, prelaunch monitoring systems, and forecast capabilities available to support the development and operations of aerospace vehicles.

Prior to the aerospace era, the early processes for acquiring measurements of the atmosphere, providing descriptions and models, and integration of this information were primarily intended to meet the needs for weather forecasting in general and to support civil and military aircraft design and operations. To some degree the processes were the product of cost-risk trade-offs and experiences associated with providing these services. Many lessons about the importance of better atmospheric information were learned from these interactions and more was learned about the atmosphere from ground based, balloon borne, and aircraft measurements. Some lessons were also derived from operational demands at the time. Examples include the inability, and thus the need, to forecast condensation trails from military aircraft, jet stream wind flow locations and magnitude, aircraft icing, etc., all the result of lessons leaned from aircraft encountering these conditions. With the advent of satellite based visual imagery and data from IR and microwave instruments, including ground based remote sensing measurements, we encountered another set of lessons to leam. They involved trying to relate these atmospheric observations to in situ measurements from ground, balloon, or aircraft sensors on which we had built a frame-ofreference" for our models and atmospheric knowledge. All of these measurements have their own unique characteristics. However, through a series of efforts in interpreting and relating these measurements, an integrated understanding of the atmosphere as required for the various applications eventually developed.

With the advent of large and relatively complex rocket systems such as the Jupiter, Atlas, Titan, and Saturn, all very expensive in terms of cost and operations for a single flight relative to aircraft, considerable effort was expended by NASA and the Air Force to maximize the value of atmospheric inputs in order to minimize risk and cost. Such is still the case with vehicles like the Space Shuttle and will also be with the anticipated new Crew Exploration Vehicle. As a result, specialized measurement systems like the Radar/Jimsphere were developed to acquire needed information on vertical wind structure with resolution beyond the capability of the rawinsonde system. Many small rockets were fired with measurement capabilities to gain data above the 
rawinsonde balloon limits for use in aerospace vehicle design and evaluation of flight performance. Similar experiences occurred with the launch and operations associated with various satellite systems. Issues concerning radiation, meteoroids, and orbital drag and control effects developed. During this period the lessons learned contributed to the advancement of knowledge of the atmospheric and space environment in varying degrees, some even benefiting other areas of atmospheric and space environment applications. Included in this scope is the more recent development of Space Weather forecasting capabilities.

In NASA, major flight programs prefer to have a Program specific natural environment design requirements document maintained under their control for efficiency in engineering studies/assessments and also for contract control reasons. As a source document and also for small flight programs, plus many other applications, a design use focused and generic definition document, such as the current NASA-HDBK-1001 'Terrestrial Environment (Climatic) Criteria Handbook For Use in Aerospace Vehicle Development" and the predecessor editions issued since the early 1960's, fills this need. This "definition" document provides information on the terrestrial environment formulated and based on experiences from the applications to aerospace vehicle design and operations, including discussions with and requests from engineers involved in the design and operations process. Another example is the unique three-dimensional timedependent "Global Reference Atmosphere Model", initially produced to support aerospace vehicle ascent, on-orbit, and re-entry thermal design calculations. As experience was gained, new information was used to update and improve the contents for subsequent editions of these items. They provided source information for the development of specific terrestrial environment requirements for the design, development, and operations of new aerospace vehicles and associated facilities necessary to meet the desired capabilities for their assigned missions.

\section{LESSONS LEARNED IDENTIFICATION}

The NASA Technical Standards Program http://standards.nasa.gov undertook an initiative to identify lessons leamed that might be linked to technical standards plus, subsequently, an effort to develop lessons leamed datasets that can be linked to the content of classroom and electronic engineering training courses. One of the actions was to identify candidate atmospheric and space environment related lessons learned that might be expanded upon for use in this initiative. Based on the experiences of various people a number of candidate lessons leamed were identified. Several are elaborated on below. They are listed in no particular order of priority or relative significance. They illustrate the type of lessons learned encountered during the past fifty years and the importance of atmospheric and space environment to the aerospace industry.

\section{Natural Environment Design Requirements For A Program: Control And Single Focus Contact}

Background: All space vehicle (launch vehicle and spacecraft) programs and projects are involved with flight through the natural (atmospheric and space) environments. There are usually several groups; both industry and government, involved in the design and development of launch vehicles and spacecraft. Having a coordinated and controlled set of natural environment design inputs tailored to meet the mission requirements for the space vehicle is critical, not only from a risk and cost aspect, but from a technical view to ensure consistent engineering analysis. Otherwise, the various trade-off analyses for the vehicle structure, control, thermal, concepts, etc. will not be based on a common terrestrial environment design requirements baseline. This will result in non-uniform products that greatly complicate the comparisons and management decisions that must be made.

Analysis: A couple of examples illustrate the importance of this lesson. (1) For the Apollo Block I/II development a contractor was provided a report containing general statistics for in-flight winds over Cape Canaveral without the required specific design wind values being specified for use. This lack of direction resulted in an extensive re-do of the analyses and design work to meet the vehicle mission requirements. (2) Early in the design trade-off studies, a Space Station contractor 
utilized an in-house orbital altitude density model for on-orbit analysis that differed from the NASA model specified for use. This resulted in different calculations for drag, re-boost, and control by the various groups involved. The recognition and establishment of a single control point for natural environment definitions and design requirements resolved both of these issues. This provided the added benefit of a common source for interpretation of the natural environment requirements.

Lesson: The specification, control, and control of natural environment definitions and requirements are important for engineering studies used in vehicle design trade-offs and development activities. This is especially true when there are several organizations involved in the vehicle development activities. It is critical that a single control point be established for natural environment inputs to ensure consistent engineering analysis and interpretation of results regarding the natural environment affects. One should note that although natural environment definitions may be the same, the design requirements may be very different for manned vehicles than those for unmanned or robotic missions.

\section{"Critical Discipline Area" Designation For Program Development}

Background: Essentially all programs, early in their development, designate critical discipline areas from which inputs are required to support the specification of program guidelines, mission requirements, and system design, Often the natural environments were not included in the list of critical disciplinary areas.

Analysis: The natural environments act on the entire aerospace vehicle system. They are usually a key driver as to what can be done and how much it will cost. Judgments must be made on how to handle the definition and requirements of the natural environments to ensure a robust design and effective use of operational procedures to mitigate environmental threats. Separation of mission critical and function critical requirements is often useful. Knowledge of (and confidence in) the distribution functions that describe environments varies greatly with the specific natural environment and the orbit or launch site. This knowledge needs to be related back to total mission risk, feasibility, and cost.

Most aerospace contractors have only limited natural environments analysis and assessment capability, with few ties between their environments specialists and their systems engineering teams. Even when the government wishes to take a "hands-off" policy, providing minimal definition of design requirements in order to allow maximum flexibility for trade-off studies, etc., the development of natural environment definitions for the mission will provide guidance for the essential trades and options that need to be examined. The involvement of natural environment specialists in formulating the specific program ground rules on risk and intensity of severe environments that must be considered will help ensure that the obstacles to mission success are addressed in these studies, thus saving a lot of time and funds for both the contractors and government program office.

Lesson: The natural environment definitions and requirements are usually one of the key drivers for the development of an aerospace vehicle program relative to accomplishment of its assigned mission. Thus, to avoid oversight of these inputs early in the establishment of program requirements, the natural environment should always be designated in the initial listing of critical disciplines for the program.

Each program systems engineering office should have a "skills checklist" and routinely review government and contractor capabilities to assure all necessary expertise is available and tied in appropriately. Care should be taken to include the lower profile but essential specialty engineering areas, including natural environment. When gaps are identified action should be taken as appropriate to bring in government or contractor specialists, establish working groups, or otherwise mitigate the problem. 


\section{One Environmental Effect Can Greatly Complicate Solution of Another}

Background: As a cost saving measure early in the program, the instrument package for the Chandra (then AXAF) was reduced and the orbit was changed from low earth orbit to high elliptical. This enabled the thermal control concept to be simplified to a passive "cold biased" surface plus heaters. This change in thermal control concept resulted in the mandating of silver backed Tefion thermal control surfaces which, in tum, had important implications for vehicle performance in the plasma environment associated with the new orbit.

Analysis: The Teflon thermal control surfaces plus the high elliptical orbit resulted in a serious spacecraft charging and electrostatic discharge environment. This necessitated an aggressive Electrostatic Discharge (ESD) test and circuit protection effort at a cost of approximately a million dollars.

Lesson: Design solutions for one environment effect can greatly complicate design solutions for other problems, including other environment related design issues. Be wary of orbit modifications that change natural environment effects on spacecraft and instruments.

Natural Environments Need To Be Considered In The Probabilistic Risk Assessment (PRA) Process

Background: A popular trend in developing top-level requirements for new aerospace vehicle programs is to include "probability of mission success" or similar criteria that are to be verified by a probabilistic risk assessment (PRA) approach. A major defect often found in this approach is the failure to consider the effects of the natural environment on the system. If a traditional PRA structure is maintained, the usual practice of excluding natural environmental effects in the risk analysis will be followed with a detriment to the system design process. The structure of the PRA process needs to be changed because the space and terrestrial environments are handled differently in a risk assessment than other failure modes. This is illustrated by some probabilistic risk assessment on-line course charts where "Failures are usually identified as initiating events." However, when failures are caused by natural environment conditions and events, the probability of the failure is very dependent on the probability of the natural environment event occurring. An example would be the occurrence of a solar proton event. The time of its occurrence and the magnitude of the resulting flux are both very probabilistic. Thus, parts failures due to radiation effects are very different than failures due to the statistical failure of parts that are operated within their specifications.

Returning to the example, we encounter the statement, "Mitigating events are events or circumstances that can prevent the undesirable event from occurring once the initiating event has occurred." Initiating events are defined as "...events that cause a perturbation in a system parameter which can cause an undesirable event (or consequence) to occur." "Failures are usually identified as initiating events." When one is undertaking a hardening program, mitigations most often occur before an initiating event, i.e. failure, not just after an initiating event as indicated in the Probabilistic Risk Assessment on-line course. In the PRA on-line course the sequence appears to be initiating event >mitigating event> undesirable event circumvented. With natural environmental issues the sequence may also be that the mitigating action prevents the initiating event from occurring in the first place.

Analysis: The probabilistic risk assessment (PRA) process example was taken from an online class. This on-line course was set up by a systems and mission analysis team and presented by their contractor to provide the basics of a program's way for performing PRA. During the review of the material it became clear that since the PRA process is a design driver, its 
lack of consideration of the effects that the various terrestrial and space environments have on the risk would be a major deficiency.

Lesson: Either the probabilistic risk assessment (PRA) process must include the natural environments effects on the system, or the limitations of the PRA process must otherwise be compensated for in the assessment. A proactive and ongoing risk review process that includes the natural environment aspects and recommends actions to the program manager is necessary to control and accept safety risks.

Unique Relationship Between Natural Environment Requirements, Other Vehicle Desian Requirements, and Vehicle Operational Requirements.

BACKGROUND: Example--The environmental constrains and flight rules for aerospace vehicle operations must be different from, but related to, the natural environment design requirements and technical constraints. In the operational realm environment monitoring, forecast, mission optimization and risk avoidance become the norm. These activities require very different environment data sets, models, and working criteria. Therefore, it becomes very important that the natural environmental risks and constraints be book-kept separately from engineering failure risks and added-on after the analysis of design factors internal to the vehicle. This will enable the vehicle to be considered as a stand-alone capability, which can be assessed later against the (different) operational natural environment factors. By taking this action, it ensures a viable and robust operational vehicle capability that will accommodate the vehicle mission operational requirements. Otherwise a vehicle will be produced that will have a lower than expected operational capability.

LESSON: Do not design an aerospace vehicle with the required design natural environment definitions and requirements incorporated and "root sum squared" as part of the other nonnominal inputs to the launch vehicle or spacecraft design. Natural environmental risks and constraints must be maintained logically and analytically separate so that accurate assessments against the operational natural environments can be made at a later date.

\section{Launch Availability With Respect To Abort Landing Site Weather}

Background: The Orbital Space Plane Program had a requirement that necessitated calculating a probability of launch availability with respect to abort landing site weather. When the contractors' proposed program configurations shifted from lifting bodies to capsules, the resulting loss of cross-range and down-range capability required that all points along the ascent trajectory be considered possible abort landing sites. Thus, instead of a few discrete locations with available long-term weather data, a continuous set of locations is required, many over the ocean with little or no long-term weather monitoring data available. The original requirement did not explicitly state how the probability was to be calculated, and at the systems design review presentations, it became apparent that the prime contractors had not adequately considered this issue. The complexity of the calculations and, more importantly, the issue of data availability, contributed to this factor not being considered in the decisions leading to the capsule concepts.

Analysis: An analysis of this situation reveals that one should state clearly in the given program requirements how the probability of launch calculations are to be performed and be aware that for a number of natural environment factors adequate data ma not be available to fully define probability distributions or confidence limits. This issue is closely related to the PRA lesson learned discussed above.

Lesson: Whenever a program requirement is written that depends on the probability of occurrence of natural environmental phenomena or of a particular set of conditions, the requirement needs to be very explicitly stated as to how the probability is to be computed, what data and models are to be used, etc. 


\section{ADDITIONAL CANDIDATE LESSONS LEAPNED SUMMARIZED}

1. TITLE: Metric-English Units Application Understanding.

BACKGROUND: Example--Radiosonde measurement calculations from the launch site used incorrect units for mean sea level. These measurements were used to calculate vehicle responses for use in flight evaluation analyses, leading to a mismatch with flight data. Mars Rover experience is another example of the importance of verifying the units used in performance calculations. SI (metric) system has been used in the scientific and international communities for many decades. More and more data sets and technical models needed in the engineering process are only available in metric units. The aerospace engineering community needs to accelerate its transition to metric units to alleviate this technical and cost issue.

LESSON: The incorrect application of units to an application can result in considerable opportunity for technical data interpretation errors and operational consequences. This is particularly true for programs that use mix of Metric and English units. Double-checking of units being used is critical to avoid issues associated therewith.

\section{TITLE: Wind Vectors Vs Engineering Vector Conventions.}

BACKGROUND: Example - Flight mechanics use of wind vectors relative to the conventional meteorological usage. In the case of flight mechanics, the vector is stated relative to direction a force is being applied. However, for meteorology, the wind vector is stated relative to direction from which wind force is coming.

LESSON: The proper interpretation and application of wind vectors is important to avoid a 180 degrees error in the vehicle's structural loads and control system response calculations.

\section{TITLE: Design Requirements, Not Climatology.}

BACKGROUND: Example-While based on climatology and models, both physical and statistical, natural environment requirements are part of the overall vehicle design effort necessary to ensure mission operational requirements are met. Thus they must be selected and defined on this basis. Simply making reference to climatological databases of atmospheric and space environment measurements will not produce the desired vehicle performance. This was done with respect to an action for the Apollo Block $\mathrm{l} / \mathrm{II}$ spacecraft and produced a costly re-design situation.

LESSON: Members of the natural environment group assigned as the control point for inputs to a program must also be part of the requirements development process. Likewise, they should be an integral part of the vehicle design team and participate in all reviews, etc. to ensure proper interpretation and application of natural environment definitions and requirements relative to overall space vehicle (launch vehicle and spacecraft) design needs.

4. TITLE: Engineering Knowledgeable Natural Environment Person to interpret Inputs for Design.

BACKGROUND: Example-Expensive icing re-analysis was required on the Shuttle External Tank. This was due to the contractor trying to sort out what information to use from an atmospheric environment database obtained from NOAA archives without the proper support and interpretation required for the specific External Tank analysis.

LESSON: The interpretation of natural environment definitions and requirements for engineering applications requires someone with both disciplinary and aerospace vehicle engineering experiences. This is important to ensure the proper selection and application of natural environment information relative to interpretations for engineering usage. 
5. TITLE: Maintain Good Records On All Natural Environment Inputs, Deviations, Interpretation, and Rational.

BACKGROUND: Example-A Skylab early re-entry issue developed relative to the natural environment requirements versus the ballistic factor estimate used for the design lifetime decision. A similar issue developed regarding the Shuttle Challenger on-pad design ambient temperature. Having the records to validate the natural environment definitions and requirements were important relative to the investigations that developed on vehicle loss accountability, etc.

LESSON: From initial studies through design, development, and operations, the responsible natural environment group must maintain complete records on all atmospheric and space environment inputs relative to basis, specification and interpretation of values for engineering use.

6. TITLE: Early Input of Natural Environment Requirements Based On Interpretation Of Mission Purpose and Operational Expectations.

BACKGROUND: Example-Develop natural environment definitions and requirements for a program as soon as practical after definition of the level one requirements for the program's mission. Thus, all concerned with the development will have a common base with associated control on changes made to natural environment definitions and requirements that impact vehicle capability or operations.

The most frequent cause of launch delays, for both the Space Shuttle and expendable vehicles, is atmospheric and space weather. Therefore, early in concept development, and repeatedly as the vehicle matures, the constraints relative to safe operations in the natural environment should be assessed based on the capability of the vehicle. This is the case for launch, on-orbit, and deep space aspects of the mission. An Atmospheric/Space Environment Parameter Analysis Model can be especially helpful in this regard. The resulting operational risk information should be incorporated into the development of the full life-cycle cost estimates and model for the vehicle program.

LESSON: The early establishment of a common set of natural environment requirements for the design and development of a vehicle is important for all concerned. This provides visibility to all, especially the program manager and systems engineers, on the operational impact of the natural environment design requirements, and ensures a consistent engineering analysis. Consideration needs to be given to the acceptable atmospheric and space environment operational constraints for space vehicle launch and spacecraft operations when developing full life-cycle cost estimates and models. An Atmospheric/Space Environment Parameter Analysis Model makes a valuable decision making tool and should be utilized in making trade-off engineering design decisions where the desired operational natural environment is a factor.

\section{TITLE: The " $1 \%$ Risk With $100 \%$ Confidence Of Not Being Exceeded" Mentality}

BACKGROUND: Example-During the Vertical Assembly Building (VAB) design wind loads requirements development, a senior person involved with the program management effort made a comment such as indicated by this lessons learned title. Once the limitations of the winds database frame-of-reference and the physical meaning of the design winds criteria was understood, a reasonable design requirement with less than $100 \%$ confidence was accepted. The clincher was comparison of design wind loads criteria for his home versus the VAB. Most natural environment phenomena do not have concretely defined extreme limits, i.e. it is always possible one will encounter a strong wind or more severe solar flare than previously observed. All rational natural environment design inputs have some degree of risk for being exceeded and this must be recognized and appreciated by all concerned. 
LESSON: The close interaction between the natural environment group and those responsible for the engineering design effort is important to ensure proper interpretation and understanding of the natural environment design requirements and associated risks.

8. TTLE: Reputation Of Natural Environment Group Must Be Developed And Maintained Through Professional Involvements And Activities Within Both The Engineering And Scientific Communities

BACKGROUND: Example-Both professional journal and technical report publications are of considerable importance in this regard. Participation in conferences, seminars, briefings, working groups are key to this matter. Awareness opportunities are necessary to ensure understanding of the natural environment definitions for engineering applications.

LESSON: The group responsible for the natural environment definitions and requirements must maintain their professional status and recognition within a dual disciplinary community (engineering and environmental science). This ensures awareness of the state of the art, respect for talents of group and their products, and maximum benefit from their work to both communities.

9. TITLE: Follow-up On Natural Environment Definitions and Requirements For Engineering Applications And Interpretations

BACKGROUND: Example-Follow-up on the Saturn vehicle design by the responsible natural environments group during design played an important role in the Saturn/Apollo lightning strike incident relative to the Satum vehicle-bonding requirement. This resulted in the issue of bonding requirements for the structure being re-addressed. Had the Saturn/Apollo vehicle been on spacecraft control it may have been lost due to lack of adequate spacecraft structure bonding.

LESSON: The responsible natural environments definitions and requirements group must exhibit the necessary initiatives to follow-up on the application and interpretation of natural environment design requirements by the engineering group users. This will serve the interests of both groups and especially help ensure the program's mission accomplishment.

10. TITLE: Ground Winds Identification And Reference For Aerospace Vehicle Design And Operations

BACKGROUND: Example - Monitoring peak ground winds is much easier to realize and visualize for design requirements and operational capability than steady state winds (that depend on the integration interval used) with a design gust value taken into account accordingly. Also, a common reference height is critical for consistency in monitoring and interpretation relative to design requirements. During the early days of operations at KSC, confusion between contractors and NASA KSC, MSFC, and JSC on this subject led to the selection of a common $18.3 \mathrm{~m}$ reference height for ground wind statements for design and operations.

LESSON: Providing a common reference height, where appropriate, for applicable natural environment statements will ensure minimum risk in engineering interpretations and operational applications.

11. TITLE: Vehicle Effects On Natural Environment Must Be Addressed Early And Action Taken To Assess And Resolve Actual Or Perceived Consequences

BACKGROUND: Example-The Shuttle solid rockets toxic exhaust by-products at launch and subsequent public reactions at the Cape are illustrative of this lesson. Also, a public ozone depletion scare concerning the stratosphere developed relative to Shuttle solid rocket exhaust until an assessment and awareness initiative assured public that it was not a threat. 
LESSON: Potential environment impacts, whether real or imaginary, must be addressed early in the development of an aerospace vehicle program. The results need to be made readily available to the public in language all will understand. Follow-ups to all public inquiries and statement, especially negative, need to be made promptly with adequate engineering and scientific documentation. It is also a matter of law that environmental impact issues be assessed prior to any major commitment of funds for a new program.

12. TITLE: Ability To Test Planned New or Changes In Natural Environment Requirements Versus Engineering Results Is Important Before Implementing Them As Formal Requirements

BACKGROUND: Example-Preliminary assessment of natural environment definitions and requirements must first be accomplished in collaboration with a responsible engineering group with respect to effect on engineering interpretations. Based on this information, then the appropriate natura! environment definitions and requirements can be provided, implemented, and controlled accordingly.

LESSON: To avoid problems with the engineering interpretation of natural environment definitions and requirements, the natural environments group responsible must first interact directly with an appropriate engineering group on their use and engineering application. This will ensure proper use and interpretation when they are formally implemented as part of the overall program design and operational requirements.

13. TITLE: Natural Environment Elements That Cannot Be Monitored and Avoided by Operational Decision Must Be Set at the Minimum Risk Level Possible, Consistent With Mission Capability Requirements. This Also Includes Those Natural Environment Elements Needed To Meet Safety And Emergency Situations.

BACKGROUND: Example-For an aerospace vehicle launch, most on pad and ascent natural environment elements can be monitored and thus taken into account before a launch decision is made. The same is true for a few on-orbit and deep space spacecraft operational requirements. In such cases, less robust design for the natural environment may be allowed, consistent with the mission requirements, along with subsequent savings on cost. Vehicle assent winds through max $Q$ is an example of where higher probability (higher risk of occurrence) natural environment design requirements may be considered for a vehicle depending on the mission. However, for situations like re-entry, which occurs over a long flight path, and on-orbit operations over a long time period, monitoring and operational options are minimal. Therefore, a robust design and acceptable minimum risk approach must be utilized.

LESSON: It is necessary to carefully analyze the mission requirements relative to an aerospace vehicle's operations and provide the required natural environment definitions and requirements accordingly. This should be accomplished in collaboration with the vehicle program manager to ensure understanding of the operational risk and full life cycle cost implications of the natural environment design requirements, both for atmospheric and space flight regimes.

14. TITLE: Reference Period For Natural Environment Definitions And Requirements Relative To Launch And On-Orbit Operations.

BACKGROUND: Example--Launch operations costs are usually significant, so it is important that launches occur in a timely manner. Hopefully, within a few days of the planned date. Thus, for launch related statements on atmospheric environment definitions and requirements, the worst reference month should be used. This provides an operational capability for the atmospheric environment that ensures that for any given month, the desired operational capability will be met or exceeded. Thus, for the worst month reference period, the maximum acceptable risk of launch delay due to natural environment will occur with other months having less probabilities of launch delay. A corresponding situation exists for space environments associated with on-orbit operational capability and deep space operations. For these cases, the anticipated lifetime in 
these operational space environment conditions must be taken into account along with the acceptable risk for comprising the mission timeline.

LESSON: All launch atmospheric environment definitions and requirements for the design of a vehicle must be made with respect to a worst month reference period. For defining the reference period for space environments associated with on-orbit and deep space operations, the anticipated lifetime in these space environment operational conditions must be taken into account along with the acceptable risks for the spacecraft's operations.

\section{CONCLUDING REMARKS}

In modem government and aerospace industry institutions the necessity of controlling currentyear costs often leads to high mobility in the technical workforce, "one-deep" technical capabilities, and minimal mentoring for young engineers. Thus, formal recording, use, and teaching of lessons leamed are especially important in the maintenance and improvement of current knowledge and development of new technologies, regardless of the discipline area. Within the NASA Technical Standards Program Website http://standards.nasa.gov there is a menu item entitled "Lessons Leamed/Best Practices". It contains links to a large number of engineering and technical disciplines related data sets that contain a wealth of lessons learned information based on past experiences. This paper has provided a small sample of lessons learned relative to the atmospheric and space environment. There are many more whose subsequent applications have improved our knowledge of the atmosphere and space environment, and the application of this knowledge to the engineering and operations for a variety of aerospace programs.

\section{ACKNOWLEDGEMENT}

This paper is based on contents of the paper "Aerospace Meteorology Lessons Learned Relative To Aerospace Vehicle Design and Operations" prepared by William W. Vaughan and B. Jeffrey Anderson for presentation at the $11^{\text {th }}$ AMS Conference on Aviation, Range, and Aerospace Meteorology, October 4-8, 2004.

The support for the preparation of this paper was provided by Paul S. Gill, Manager, NASA Technical Standards Program.

\section{BIBLIOGRAPHY}

Anon., 2000: "Terrestrial Environment (Climatic) Criteria Handbook for Use in Aerospace Vehicle Development", NASA-HDBK-1001.

Anon., 2004: “MSFC Exploration Systems Lessons Leamed Workshop Report". NASA Marshall Space Flight Center, August 5, 2004.

Johnson, Dale L., C. Kelly Hill, William W. Vaughan, S. Clark Brown, and G. Wade Batts, 1993: "Natural Environment Requirements Definition and Significance for Aerospace Plane Development". Paper \#AIAA-93-5074. AIAADGLR Fifth International Aerospace Planes and Hypersonic Technologies conference, November 30-December 3, 1993, Munich, Germany.

Justus, C.G. and D.L. Johnson, 1999: "The NASA/MSFC Global Reference Atmospheric Model 1999 Version (GRAM-99)", NASA TM-1999-209630.

Larson W. (chief editor), D. Kirkpatrick, R. Ryan and V. Weyers, pending publication: "Space Launch and Transportation Systems (SLaTS): Design and Operations" book: Chapter 7, "The Role of the Natural Environments in Launch Vehicle Development", authored by Dale L. Johnson and B. Jeffrey Anderson. 
Pearson, S.D., W.W. Vaughan, G.W. Batts, and G.L. Jasper, 1996: "Importance of the Natural Terrestrial Environment With Regard to Advanced Launch Vehicle Design and Development". NASA TM 108511.

Ryan, R., J. et al., 1996: "Working on the Boundaries: Philosophies and Practices of the Design Process", NASATP-3642.

Vaughan, W. W. and B. J. Anderson, 2004: "Aerospace Meteorology Lessons Learned Relative To Aerospace Vehicle Design and Operations". Proceedings of the $11^{\text {th }}$ Aviation, Range, and Aerospace Meteorology Conference, American Meteorological Society, Boston, MA.

Vaughan, W. W. and S. C. Brown, 1985: "Natural Environment Considerations for Space Shuttle System Development Support". Joumal of Spacecraft and Rockets, 22 (3), 355-360.

Vaughan, W.W., D.L. Johnson, S.D. Pearson and G. W. Batts, 1997: "The Role of Aerospace Meteorology in the Design, Development and Operation of New Advance Launch Vehicles". Proceedings of the Seventh Conference on Aviation, Range and Aerospace Meteorology, American Meteorological Society, Boston, MA.

Vaughan, W.W., L.J. Ehemberger and D.L. Johnson, 1999: "An Overview of Atmospheric Modeling for Aeronautical and Aerospace Vehicle Simulation Applications", Paper \#99-4188 presented at AIAA Modeling \& Simulation Technologies Conference, Portland OR 9-11 August 1999.

Prepared October 22, 2004 